Ann. Biol. anim. Bioch. Biophys., I965, 5 (2), 267-281.

\title{
LE MÉTABISULFITE DE POTASSIUM ÉTUDE DE TOXICITÉ A LONG TERME SUR LE RAT
}

\author{
R. CluzAN, J. CAUSERET et Denise HUGOT \\ Laboratoire d'Anatomie pathologique, Hôpital Saint-Michel, Paris \\ Laboratoire d'Étude des Qualités biologiques des Aliments de l'Homme, \\ Centre national de Recherches zootechniques, Jouy-en-Josas (Seine-et-Oise)
}

\section{SOMMAIRE}

Pendant 20 mois, deux lots de 80 rats (40 mâles et 40 femelles) ont été soumis à un même régime alimentaire équilibré et ont reçu respectivement, à titre de boisson, soit de l'eau distillée, soit une solution de métabisulfite de potassium à $\mathrm{I}, 2 \mathrm{~g}$ par litre.

Aucune différence n'a été observée entre les deux lots, en ce qui concerne la mortalité, l'évolution pondérale et la consommation de nourriture, les manifestations cliniques, les formules sanguines (sauf en ce qui concerne le nombre des leucocytes), le poids de différents organes (sauf en ce qui concerne celui de la rate), et l'étude anatomique - macroscopique et microscopique - de tous les viscères prélevés à l'issue de l'expérience (cesophage, estomac, grêle, côlon, foie, reins, cœur, poumons, rate, pancréas, surrénales, gonades, fragments de muscles, encéphale, trachée).

Les seuls effets particuliers notés dans le lot d'animaux qui a reçu du métabisulfite de potassium sont : une augmentation du nombre des leucocytes (significative chez les mâles seulement) et une augmentation du poids de la rate (chez les femelles seulement).

D'autre part, au cours de deux reproductions successives, on a observé, dans le lot qui recevait du métabisulfite de potassium, une diminution du nombre moyen des jeunes par portée et de la proportion des mâles dans les portées, par rapport aux résultats obtenus dans le lot témoin. Cependant, la croissance des jeunes - soumis eux-mêmes au régime de leurs parents - a été identique dans les deux lots durant trois mois de détermination.

Le caractère limité des différences observées entre les deux lots et les difficultés de leur interprétation ne permettent pas de conclure actuellement à la nocivité du métabisulfite de potassium dans les conditions où il a été administré. Des recherches complémentaires seront indispensables, en particulier dans le domaine de la reproduction.

\section{INTRODUCTION}

L'anhydride sulfureux et quelques-uns des sels qui en dérivent (notamment le métabisulfite de potassium) figurent parmi les antiseptiques les plus anciennement et les plus largement employés en technologie alimentaire (TRUHAUT, I955 et I962). En France, on connait en particulier leurs utilisations en cenologie, en cidrerie, 
en brasserie, dans l'industrie des jus de fruits, pour la conservation des poissons séchés, des crevettes roses, des fruits secs, des pommes de terre grattées, etc. (TRUhaut et Souveraix, Ig63).

Cependant, les effets physiopathologiques de l'ingestion de sulfites font encore. l'objet de diverses incertitudes, dues au petit nombre des observations expérimentales approfondies et aux résultats discordants des travaux publiés.

La plupart de ces travaux datent de plus d'un demi-siècle. Le plus ancien est dût à LEUCH (I895), qui a administré à une trentaine de personnes diverses des doses de $\mathrm{SO}_{2}$ dans une petite quantité de vin $(30$ à $50 \mathrm{ml})$; il observa des troubles divers (irritation de la gorge, brûlures d'estomac, migraines) pour les doses de $\mathrm{SO}_{2}$ libre égales ou supérieures à $45 \mathrm{mg}$. WALBAUM (I906) a également signalé une irritation du tractus digestif chez des sujets qui avaient reçu quatre fois par jour, pendant quatre jours, des doses de sulfite de sodium comprises entre 3 Io et $620 \mathrm{mg}$. D'autre part, WILEY (I907) a étudié le problème chez douze sujets qui avaient reçu pendant

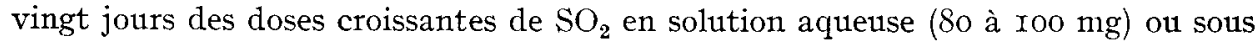
forme de sulfite de sodium en capsules (I I 5 à $760 \mathrm{mg}$ ) ; il signala - outre des symptômes subjectifs tels que céphalées, étourdissements, sensations de faiblesse - des troubles rénaux et une anémie hypochrome. Il en conclut à la nécessité de proscrire $\mathrm{SO}_{2}$, sous toutes ses formes, de toutes les denrées alimentaires.

A la même époque, quelques travaux ont été effectués sur le Chien. KIonka (I896) et Schulz (I9O2) ont observé des effets nocifs - en particulier des hémorragies - après administration prolongée de faibles doses de sulfites. En revanche, Wildenrath (Igo6), LehmaNn et Treu'Tleis (Igog), Ros't et Franz (I9I3) ont conclu à l'innocuité, pour le Chien, de doses de $\mathrm{SO}_{2}$ allant jusqu'à roo et même $200 \mathrm{mg}$ par $\mathrm{kg}$; les derniers auteurs cités ont fait subir ce traitement à leurs animaux pendant un an sans voir apparaître aucune lésion macroscopique ou microscopique.

Les conclusions de certains de ces travaux - et singulièrement celles des observations sur l'Homme - ayant ému les milieux viticoles, une Commission scientifique fut constituée en IgIo, à Bordeaux, pour reprendre l'étude du problème dans ses aspects œnologiques. Sous l'impulsion de cette commission, une première série de recherches fut effectuée sur des chiens qui reçurent quotidiennement, durant un mois, Io $\mathrm{ml}$ par $\mathrm{kg}$ de poids, d'un vin contenant Ioo $\mathrm{mg}$ de $\mathrm{SO}_{2}$ libre ou $400 \mathrm{mg}$ de $\mathrm{SO}_{2}$ total (dont $300 \mathrm{mg}$ à l'état combiné) par litre. En aucun cas, on n'observa de troubles gastriques, rénaux ou sanguins ; à l'autopsie, tous les organes paraissaient normaux (GAUTRELET, IgIo $a$ et IgIo b).

Une seconde série d'expériences fut tentée sur six hommes qui, pendant plusieurs semaines, ingérèrent chaque jour 15 à $20 \mathrm{ml}$ par $\mathrm{kg}$ de poids, d'un vin renfermant $400 \mathrm{mg}$ de $\mathrm{SO}_{2}$ par litre (dont $300 \mathrm{mg}$ à l'état combiné) ; aucun sujet ne fut atteint de troubles rénaux ou sanguins (GAUTRELET, IgIo $a$ et IgIo $b$ ).

Bien que la durée de ces expériences ait été trop courte pour conduire à des résultats probants, il a fallu attendre près de 40 ans pour voir apparaître de nouvelles publications sur le même problème. En I946, FitzhuGH, KNUDSEN et NELSON ont publié le compte rendu d'une étude de toxicité à long terme sur des rats dont le régime contenait de 0,05 à 2 p. Ioo de métabisulfite de sodium (soit 0,03 à $I, 2$ p. Ioo de $\mathrm{SO}_{2}$ ). Pour les doses de métabisulfite égales ou supérieures à 0, Io $p$. IOO, ils signalent un ralentissement de la croissance et le développement de troubles macroscopiques et microscopiques: "yeux à lunettes ", crises de polynévrite, atrophie de 
différents organes, calcifications rénales, fibrose et hypertrophie épithéliale gastrique "squameuse ", altérations de l'utérus, dépigmentation des incisives, etc. Une partie de ces phénomènes pouvait s'expliquet par une carence en thiamine : on sait en effet que cette vitamine est détruite en présence de sulfites. Toutefois, l'administration d'un supplément de thiamine laissait subsister la plupart des troubles et des lésions, les crises de polynévrite étant seules à disparaître.

En 1955, nous avons effectué, en collaboration avec FLANZY, une étude de caractère préliminaire sur les effets respectifs, chez le Rat, d'un vin de Muscadet naturel, du même vin sulfité à l'avance par addition de $400 \mathrm{mg}$ de métabisulfite de potassium par litre ( $25 \mathrm{p}$. Ioo de $\mathrm{SO}_{2}$ ainsi ajouté passant à l'état combiné), et du même vin sulfité à la même dose au moment même de l'administration. Les sujets qui, pendant 6 mois, recevaient les vins sulfités, ont pris du poids moins rapidement que ceux qui recevaient le vin naturel; c'est le vin sulfité au moment même de 1'emploi qui a entraîné le ralentissement de la croissance le plus marqué. En outre, ce vin a provoqué une nette hypertrophie du foie.

En revanche, HöTzEL. (Ig6I-I962) n'a observé aucune modification du gain de poids, de l'indice de consommation et du poids de différents organes chez le Rat soumis pendant roo jours à un régime alimentaire équilibré, additionné de doses de sulfites correspondant à $400 \mathrm{mg}$ de $\mathrm{SO}_{2}$ par $\mathrm{kg}$ de poids corporel.

Enfin, alors que l'étude qui fait l'objet de ce mémoire prenait fin, une communication sur le même sujet a été présentée par LanTeaume et al. (I965) devant la Société des Fxperts-Chimistes de France. Ces auteurs ont administré quotidiennement par intubation gastrique à divers lots de rats, pendant 2 ans, $3 \mathrm{ml}$ pour roo grammes de poids de l'un des liquides suivants:

- eau pure;

- solution aqueuse de métabisulfite de potassium contenant $45^{\circ} \mathrm{mg}$ de $\mathrm{SO}_{2}$ par litre ;

- vin rouge de coupage contenant rIo $\mathrm{mg}$ de $\mathrm{SO}_{2}$ par litre;

- même vin contenant $450 \mathrm{mg}$ de $\mathrm{SO}_{2}$ par litre.

L'anhydride sulfureux n'a provoqué ni modifications de la croissance, ni troubles de la reproduction, ni lésions organiques décelables macroscopiquement ou microscopiquement.

\section{PROTOCOLE EXPÉRIMENTAI}

Les méthodes d'étude mises en œuvre s'inspirent largement du protocole expérimental recommandé par la F.A.O. (1958) pour l'étude toxicologique des additifs chimiques et sont proches aussi de celui qui a été décrit dans une récente circulaire du Ministère de la Santé Publique et de la Population (1963).

\section{Animaux}

L'étude a porté sur 160 rats Wistar ( 80 mâles et 80 femelles), provenant de l'élevage du laboratoire. Au début de l'étude, ils étaient âgés de 28 à 32 jours et pesaient de 50 à 60 grammes. Leur état sanitaire pouvait être considéré comme satisfaisant (courbes de croissance conformes à celles obtenues habituellement au laboratoire, pas d'infection apparente).

Tous ces animaux ont été soumis à un régime alimentaire complet et équilibré. Mais la moitié d'entre eux a reçu comme boisson de l'eau distillée (lot I) et l'autre moitié une solution de métabisulfite de potassium à I,2 gramme par litre, soit environ $700 \mathrm{mg}$ de $\mathrm{SO}_{2}$ par litre (lot 2). 


\section{Régime alimentaire}

Les animaux ont été soumis pendant la durée de l'expérience à un régime alimentaire équilibré :

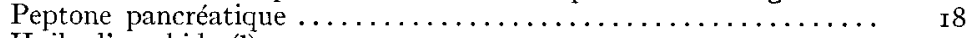

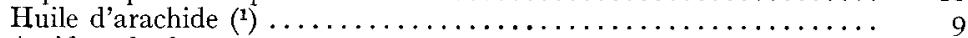

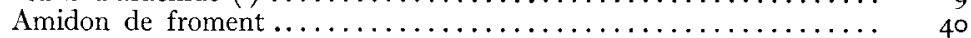

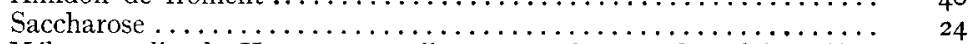

Mélange salin de HubBel et coll., sans carbonate de calcium $\left({ }^{2}\right) \ldots \quad 2$

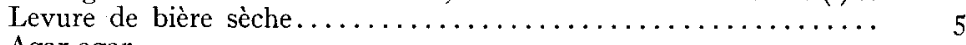

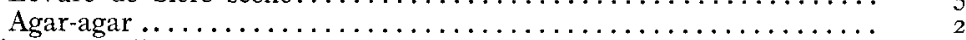

Ce régime a été distribué sous forme de bouillie obtenue par addition de $35^{\circ} \mathrm{ml}$ d'eau distillée à I $\mathrm{kg}$ du mélange précédent.

\section{Administration du métabisulfte de potassium}

Le mode d'administration du métabisulfite de potassium (en solution dans l'eau de boisson) a été choisi en vue d'éviter la destruction partielle de la thiamine du régime par $\mathrm{SO}_{2}$ avant l'ingestion. Malgré l'odeur et la saveur désagréables de cette solution, - nettement perceptibles pour le consommateur humain -, les rats l'ont acceptée sans difficulté (tabl. I).

TABLEAU I

Consommation de boisson des animaux d'expérience

\begin{tabular}{|c|c|c|c|}
\hline & \multicolumn{3}{|c|}{$\begin{array}{l}\text { Consommation moyenne de boisson } \\
\text { (ml par jour) }\end{array}$} \\
\hline & $1^{\mathrm{er}}-15^{\mathrm{e}}$ jours & $2^{e}$ mois & $\begin{array}{l}\text { A partir } \\
\text { dul ge mois }\end{array}$ \\
\hline Lot 1 (eau) & & & \\
\hline Mâles ............... & 16,2 & 16,0 & 18,1 \\
\hline $\begin{array}{l}\text { Femelles ............ } \\
\text { Lot } 2 \text { (solution sulfitée) }\end{array}$ & 15,3 & 16,0 & 17,8 \\
\hline Mâles...$\ldots \ldots \ldots \ldots$. & 14,8 & 16,0 & 19,2 \\
\hline Femelles ............ & 15,3 & 16,8 & 18,1 \\
\hline
\end{tabular}

Pour permettre de comparer les doses de $\mathrm{SO}_{2}$ ingérées par les rats du lot I et celles administrées par d'autres expérimentateurs, nous avons rapporté ces doses aux quantités de nourriture consommées et au poids corporel (tabl. 2).

Les différences liées au sexe résultent du fait que les femelles ingèrent autant de boisson que les mâles, mais consomment moins de nourriture et prennent moins de poids.

\section{Durée de l'étude}

L'étude a commencé le I4 juin 1962, et le sacrifice des animaux a été échelonné sur la période

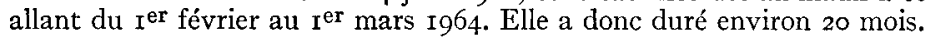

(1) Additionnée de r p. roo d'huile de foie de morue et de o,o 5 p. Ioo d' $\alpha$-tocophérol.

(2) Le calcium est fourni par les autres constituants du régime, en particulier par la peptone. 


\section{Observations}

Les observations essentielles ont porté sur les points suivants :

- mortalité,

- évolution pondérale,

- consommation de nourriture,

- manifestations d'ordre clinique ;

- nombre de portées, leur constitution, développement pondéral des jeunes (deux générations successives) ;

TABLEAU 2

Quantités de métabisulfite de potassium et de $\mathrm{SO}_{2}$ ingérées en moyenne par les animaux du lot 2

(rapportées aux quantités de régime consommé ou au poids corporel)

\begin{tabular}{|c|c|c|c|c|}
\hline & \multicolumn{2}{|c|}{ Pour $100 \mathrm{~g}$ de régime consommé } & \multicolumn{2}{|c|}{ Pour $100 \mathrm{~g}$ de poids corporel } \\
\hline & $\begin{array}{c}\text { Métabisulfite de } \mathrm{K} \\
(\mathrm{mg})\end{array}$ & $\begin{array}{l}\mathrm{SO}_{2} \\
(\mathrm{mg})\end{array}$ & $\begin{array}{c}\text { Métabisulfite de } \mathrm{K} \\
(\mathrm{mg})\end{array}$ & $\begin{array}{l}\mathrm{SO}_{2} \\
(\mathrm{mg})\end{array}$ \\
\hline \multicolumn{5}{|l|}{ Mâles } \\
\hline $1^{e r}-15^{e}$ jours $\ldots \ldots \ldots$ & 163 & 95 & 33 & 19,0 \\
\hline $2^{\circ}$ mois ............. & 149 & 86 & 10 & 5,8 \\
\hline $9^{\mathrm{e}}$ mois $\ldots \ldots \ldots \ldots$. & 157 & 91 & 6,1 & 3,5 \\
\hline $20^{e}$ mois $\ldots \ldots \ldots \ldots \ldots$ & - & - & 5,0 & 2,9 \\
\hline \multicolumn{5}{|l|}{ Femelles } \\
\hline 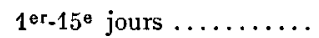 & 178 & 103 & 33 & 19,0 \\
\hline $2^{\circ}$ mois..$\ldots \ldots \ldots \ldots$ & 189 & 110 & 13,5 & 7,8 \\
\hline $9^{9}$ mois $\ldots \ldots \ldots \ldots$ & 187 & 108 & 9,3 & 5,4 \\
\hline $20^{\circ}$ mois..$\ldots \ldots \ldots \ldots$ & - & - & 7,0 & 4,0 \\
\hline
\end{tabular}

- études hématologiques sur Ioo sujets de la première génération (25 mâles et 25 femelles de chaque lot) : des numérations d'hématies et de leucocytes ont été effectuées au moment du sacrifice des animaux, et une étude complémentaire a porté sur des frottis de moelle osseuse et sur des frottis de sang, les résultats étant comparés à ceux des numérations ;

- étude anatomique des animaux de la première génération morts pendant l'expérience et des I00 sujets sacrifiés à son issue (par le gaz d'éclairage), cette étude comportant la recherche de lésions macroscopiques, la pesée de certains organes et l'étude microscopique des viscères suivants, après fixation dans le formol à $10 \mathrm{p}$. I00, inclusion à la paraffine et coloration par l'hématéine-éosine avec, à la demande, coloration élective : csophage, estomac, grêle, côlon, foie, reins, cœur, poumons, rate, pancréas, surrénales, gonades, fragments de muscles, encéphale, trachée.

\section{RÉSULTATS}

\section{Mortalité}

La mortalité en cours d'expérience a atteint :

- 9 sujets du lot 1 (témoins), dont 6 mâles et 3 femelles : soit I2 p. Ioo de l'effectif total du lot;

- II sujets du lot 2 (recevant $\mathrm{SO}_{2}$ ), dont 8 mâles et 3 femelles : soit I4 p. Ioo de l'effectif total du lot. 
Dans les deux lots, cette mortalité est due presque entièrement à une affection des voies respiratoires qui, en juin I963 a frappé brutalement l'animalerie du laboratoire, en provoquant d'ailleurs une diminution marquée de la consommation de nourriture et une chute de poids chez de nombreux sujets. Des injections intramusculaires d'oxytétracycline $(2,5 \mathrm{mg}$ p. $100 \mathrm{~g}$ de poids pendant trois jours consécutifs) ont été pratiquées sur tous les animaux sans distinction et ont enrayé les progrès de l'épidémie. La perte de poids a été compensée et l'expérience s'est poursuivie sans nouvel incident pendant les 7 derniers mois.

Si l'on défalque des valeurs citées plus haut les décès observés en juin rg63, la mortalité a été :

dans le lot $I$, de 3 sujets (I mâle et 2 femelles);

dans le lot 2 , de 3 sujets (I mâle et 2 femelles).

Il ne semble donc pas qu'il ait y lieu de lui accorder une signification particulière dans le cadre de l'expérience.

\section{Évolution pondérale}

Ne pouvant reproduire ici les courbes de poids complètes, nous nous bornerons à indiquer les gains de poids à divers stades de l'expérience (tab1. 3).

Les faibles différences observées entre les deux lots ne sont pas significatives.

TABIEAU 3

Gains de poids des animaux d'expérience

\begin{tabular}{|c|c|c|c|c|}
\hline & \multicolumn{4}{|c|}{ Gains de poids moyens $(\mathrm{g})$} \\
\hline & $\begin{array}{l}\text { Après } \\
1 \text { mois }\end{array}$ & $\begin{array}{l}\text { Après } \\
2 \text { mois }\end{array}$ & $\begin{array}{l}\text { Après } \\
6 \text { mois }\end{array}$ & $\begin{array}{l}\text { Après } \\
20 \text { mois }\end{array}$ \\
\hline Wales & & & & \\
\hline Lot 1 (eau) ........... & 88 & 154 & 275 & 398 \\
\hline Lot 2 (solution sulfitée) .. & 88 & 150 & 274 & 392 \\
\hline Femelles & & & & \\
\hline Lot 1 (eau) $\ldots \ldots \ldots \ldots$ & 6.2 & 102 & 151 & 237 \\
\hline Lot 2 (solution sulfitée) .. & 69 & $10 t$ & 151 & 213 \\
\hline
\end{tabular}

\section{Consommation de nourriture}

La consommation de nourriture a été déterminée à plusieurs reprises au cours de l'expérience, chaque fois pendant une période de 2 semaines. Aucune différence significative n'a été observée entre les deux lots.

\section{Manifestations d'ordre clinique}

En dehors de l'affection des voies respiratoires déjà signalée, un certain nombre de sujets ont présenté, au cours de la deuxième année, des tumeurs sous-cutanées volumineuses siégeant en particulier au niveau de l'abdomen (deux dans le lot I 
et deux dans le lot 2). En revanche, les crises de polynévrite et le syndrome des " yeux à lunettes " signalées par FITZHUGH, KNUDSEN et NELSON (r946) n'ont été observés chez aucun de nos animaux.

\section{Reproduction}

A 1'âge de neuf mois, un certain nombre de femelles, choisies au hasard dans chaque lot, ont été mises au mâle (dans une même cage : 3 femelles avec 2 mâles du même lot).

Les animaux de la deuxième génération ont été soumis dès le sevrage (à 2 I jours) au régime de leurs parents. Lorsqu'ils ont atteint 1'âge de trois mois, ils ont été mis en couple, et les animaux de la troisième génération ont été soumis au régime de leurs parents depuis le sevrage jusqu'à l'âge de trois mois.

Les résultats obtenus sont mentionnés dans le tableau 4 .

TABLEAU 4

Reproduction des animaux

Nombre de femelles accouplées. .

Nombre de femelles n'ayant pas donné de portée . ............

Durée écoulée entre la mise en couple et la mise bas (jours) $\left(^{*}\right)$.

Nombre de jeunes par portée $\left(^{*}\right)$.

Proportion de mâles sur l'ensemble des jeunes (p. 100) .......

Proportion des portées où les mâles sont les plus nombreux (p. 100).

Gain de poids des jeunes mâles en 3 mois $(g) . \ldots \ldots \ldots \ldots \ldots \ldots$

Gain de poids des jeunes femelles en 3 mois $(g)$

\begin{tabular}{|c|c|c|c|}
\hline \multicolumn{2}{|c|}{$\begin{array}{l}\text { Reproduction des sujets } \\
\text { de la } 1^{\text {re }} \text { génération }\end{array}$} & \multicolumn{2}{|c|}{$\begin{array}{l}\text { Reproduction des sujets } \\
\text { de la } 2^{\mathrm{e}} \text { génération }\end{array}$} \\
\hline Lot 1 (eau) & Lot $2\left(\mathrm{SO}_{2}\right)$ & Lot 1 (eau) & Lot $2\left(\mathrm{SO}_{2}\right)$ \\
\hline 22 & 21 & 23 & 20 \\
\hline 0 & 4 & 2 & 3 \\
\hline $30,7 \pm 2,2$ & $33,1 \pm 2,5$ & $25,2 \pm 0,9$ & $30,3 \pm 2,5$ \\
\hline $7,5 \pm 0,5$ & $6,1 \pm 0,4$ & $8,8 \pm 0,5$ & $7,8 \pm 0,6$ \\
\hline 52 & 45 & 53 & 39 \\
\hline 59 & 42 & 53 & 21 \\
\hline 240 & 239 & 214 & 219 \\
\hline 133 & 143 & 126 & 136 \\
\hline
\end{tabular}

$\left(^{*}\right)$ La seconde valeur citée dans chaque colonne de chiffres représente l'erreur standard de la moyenne :

$$
E=\sqrt{\overline{\Sigma(x-\bar{x})^{2}}}
$$

Il existe une différence significative entre les deux lots en ce qui concerne le nombre de jeunes par portée au cours de la première reproduction: en moyenne, il est diminué de près de $20 \mathrm{p}$. Ioo dans le lot 2 (différence significative au seuil de $P=0.05$ ). Une différence du même genre est observée au cours de la seconde reproduction, mais elle est moins marquée et non significative.

D'autre part, la proportion des mâles dans le lot 2 est moins élevée que dans

Annales de Biologie animale. - I965. 
le lot I. La comparaison des résultats obtenus dans les deux lots - par la méthode dite due $\chi^{2}$ - prouve que la différence observée au cours de la première reproduction peut être due aux hasards de l'échantillonnage, mais que celle observée au cours de la deuxième reproduction est hautement significative (au seuil de $\mathrm{P}=0 . \mathrm{OI}$ ).

Toutes les autres déterminations donnent des résultats identiques pour les deux lots.

\section{Étude hématologique}

a) Le nombre des hématies et des leucocytes contenus dans le sang des animaux à la fin de l'expérience est indiqué dans le tableau 5 .

TABLEAU 5

Numérations globulaires $\left({ }^{*}\right)$

\begin{tabular}{|c|c|c|}
\hline & $\begin{array}{l}\text { Hématies } \\
\text { (millions par ml) }\end{array}$ & $\begin{array}{c}\text { Leucocytes } \\
\text { (milliers par ml) }\end{array}$ \\
\hline Mâtes & & \\
\hline Lot 1 (eau)..$\ldots \ldots \ldots$ & $8,94 \pm 0,12$ & $8,79 \pm 0,73$ \\
\hline $\begin{array}{c}\text { Lot } 2\left(\mathrm{SO}_{2}\right) \ldots \ldots \ldots \ldots \\
\text { Femelles }\end{array}$ & $8,90 \doteq 0,28$ & $11,12 \pm 0,91$ \\
\hline $\begin{array}{l}\text { Lot } 1(\mathrm{eau}) \ldots \ldots \ldots \ldots \\
\text { Lot } 2\left(\mathrm{SO}_{2}\right) \ldots \ldots \ldots \ldots\end{array}$ & $\begin{array}{l}8,35 \pm 0,37 \\
8,25 \pm 0,31\end{array}$ & $\begin{array}{l}6,44 \pm 0,54 \\
7,17 \pm 0,52\end{array}$ \\
\hline
\end{tabular}

(*) La seconde valeur citée dans chaque colonne de chiffres représente l'erreur standard de la moyenne:

$$
\mathrm{E}=\sqrt{\frac{\sum(x-\bar{x})^{2}}{\mathrm{~N}(\mathrm{~N}-1)}}
$$

Dans le cas des hématies, la faible différence observée entre les deux lots n'est pas significative. Le taux des leucocytes est plus élevé dans le lot $2\left(\mathrm{SO}_{2}\right)$ mais la différence n'est significative que chez les mâles (au seuil de $\mathrm{P}=0.05$ ).

b) Certains animaux présentaient des anomalies de leur formule sanguine: ainsi a-t-on observé une hyperleucocytose avec polynucléose neutrophile (hyperleucocytose atteignant parfois I6 o0o à I9 000), en rapport avec des lésions pulmonaires retrouvées à l'examen anatomique. Ces animaux étaient un peu plus nombreux dans le lot $2\left(\mathrm{SO}_{2}\right)$. D'autre part, quelques animaux présentaient une leucopénie, mais ceci avec une fréquence égale dans les deux groupes.

c) L'étude des frottis médullaires n'a pas montré de différence entre les deux groupes (tab1. 6). La lecture des lames médullaires a cependant été techniquement difficile, du fait de la richesse en graisse de la moelle du Rat, ce qui a rendu délicat le problème de la coloration des lames. 


\section{TABLEAU 6}

Myélogrammes (valeurs moyennes)

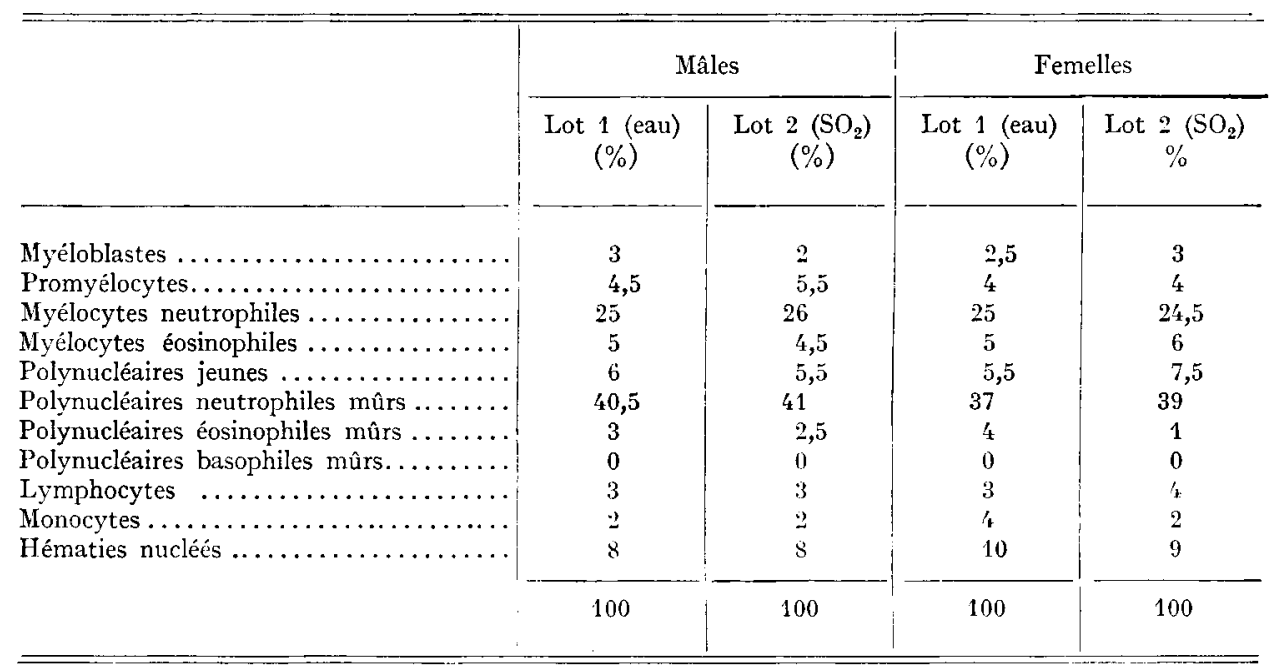

\section{Poids de divers organes}

Le poids moyen de divers organes, rapporté à celui du corps, figure dans le tableau 7 .

La comparaison des valeurs obtenues dans les deux lots ne permet de relever

$$
\text { TABLEAU } 7
$$

Poids de quelques organes

(pour $100 \mathrm{~g}$ de poids corporel) $\left(^{*}\right)$

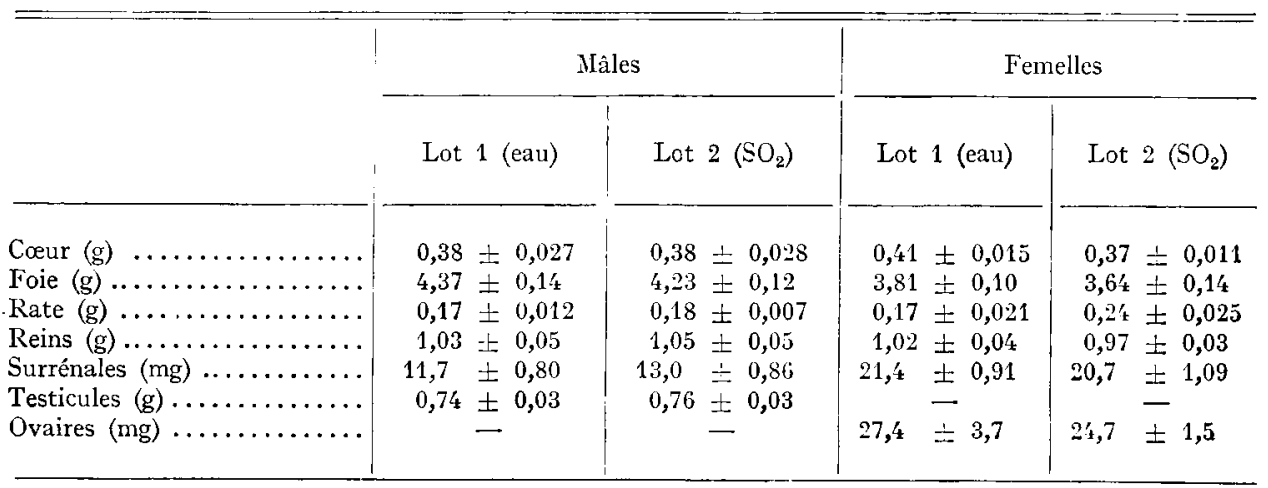

$\left({ }^{*}\right)$ La seconde valeur citée dans chaque colonne de chiffres représente l'erreur standard de la moyenne

$$
E=\sqrt{\frac{\sum(x-)^{2}}{N(N-1)}}
$$


qu'une seule différence significative : elle concerne le poids de la rate, qui est plus élevé chez les femelles du lot 2 que chez celles du lot I (différence significative au seuil de $\mathrm{P}=0.05$ ).

\section{Etude anatomique: observations macroscopiques}

Les principales observations macroscopiques sont résumées dans le tableau 8 . Bien que certaines lésions n'aient été observées que dans le lot 2 , aucune d'entre elles n'a été suffisamment fréquente pour qu'on puisse conclure à l'existence de différences entre les deux lots.

\section{TABLeAU 8}

Étude anatomique: observations macroscopiques

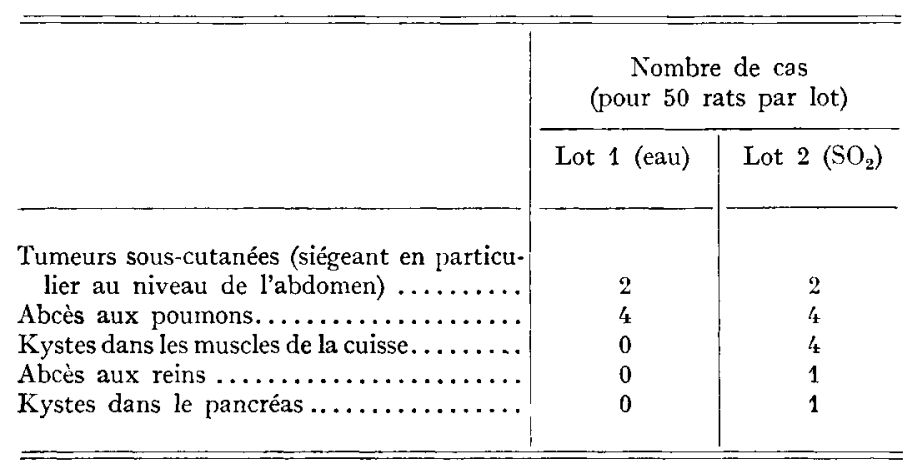

Étude anatomique: observations microscopiques

a) Au niveau du foie, ont été observées:

- une dilatation sinusale très fréquente dans les deux lots, plus ou moins marquée, à disposition centro-lobulaire, en rapport avec le mode de sacrifice sans aucun doute :

- une stéatose se répartissant comme l'indique le tableau 9.

\section{TABLEAU 9}

Répartition des cas de stéatose hépatique

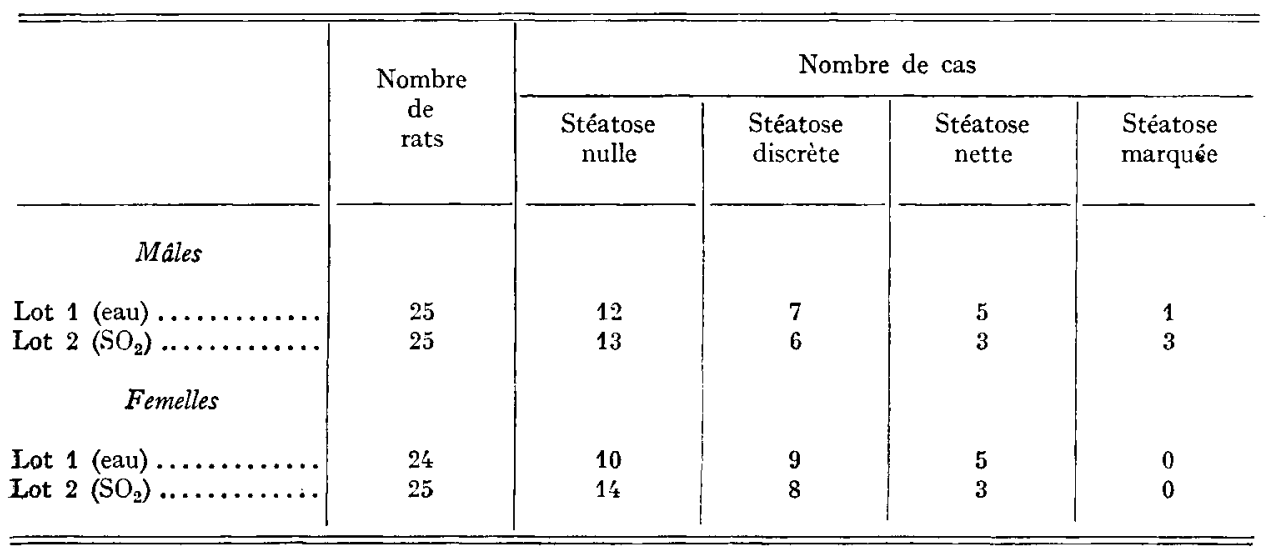


Cette stéatose, sans particularité histologique, se retrouve avec une fréquence comparable dans les deux groupes d'animaux.

b) Au niveau de l'osophage, on a trouvé, chez un mâle qui avait reçu du métabisulfite, une ulcération du bas œsophage. Cette ulcération ne peut vraisemblablement être retenue comme lésion due au sulfite, car de multiples facteurs peuvent expliquer une telle lésion chez un rongeur; d'autre part, elle a été rencontrée une seule fois.

Aucune différence n'est à signaler entre les deux lots dans la structure de la portion œesophago-gastrique et les régions voisines. Dans les deux lots, l'épithélium malpighien a paru légèrement hyperplasique dans cette région.

c) Au niveau des reins, on a trouvé parfois des cylindres hyalins, des cylindres granuleux, des dilatations tubulaires kystiques (tabl. ro). Mais la répartition de ces lésions est identique dans les deux lots.

\section{TABleau Io}

Répartition de diverses lésions rénales

\begin{tabular}{|c|c|c|c|c|}
\hline & \multirow{2}{*}{$\begin{array}{c}\text { Nombre } \\
\text { de } \\
\text { rats }\end{array}$} & \multicolumn{3}{|c|}{ Nombre de cas } \\
\hline & & $\begin{array}{l}\text { Cylindres } \\
\text { hy:alins }\end{array}$ & $\begin{array}{l}\text { Cylindres } \\
\text { granuleux }\end{array}$ & $\begin{array}{c}\text { Dilatations } \\
\text { kystiques }\end{array}$ \\
\hline Mâles & & & & \\
\hline Lot 1 (eau) ...... & 25 & 4 & 1 & 1 \\
\hline $\begin{array}{c}\text { Lot } 2\left(\mathrm{SO}_{2}\right) \ldots \ldots \\
\text { Femelles }\end{array}$ & 25 & 6 & 1 & 3 \\
\hline Lot 1 (eau) ...... & 24 & 4 & 2 & 1 \\
\hline Lot $2\left(\mathrm{SO}_{2}\right) \ldots \ldots$ & 25 & 3 & 0 & 1 \\
\hline
\end{tabular}

Quelques images de hyalinisation des basales ont été observées, mais le phénomène restait limité et se retrouvait dans les deux lots. Ces modifications sont à rattacher probablement à des phénomènes de vieillissement.

Il n'a pas été observé de calcification.

Une fois, chez un mâle du lot $2\left(\mathrm{SO}_{2}\right)$, il a été observé des images de régression tubulaire, paraissant plus en rapport avec des phénomènes infectieux que toxiques (abcès rénal constaté à l'autopsie).

d) Au niveau des poumons, de nombreuses lésions inflammatoires à prédominance péribronchique, de caractère aigu au subaigu, ont été retrouvées, en rapport avec des phénomènes cliniques signalés précédemment. Leur répartition était la suivante :

Mâles, $\operatorname{lot} I_{\text {( }}(\mathrm{eau}) \ldots \ldots \ldots \ldots \ldots \ldots \ldots \ldots \ldots \ldots \ldots \ldots \ldots$ cas

Mâles, 1 tot $2\left(\mathrm{SO}_{2}\right) \ldots \ldots \ldots \ldots \ldots \ldots \ldots \ldots$ I2 cas

Femelles, 1 ot $\mathrm{I}$ (eaur) ......................... I2 cas

Femelles, lot $2\left(\mathrm{SO}_{2}\right) \ldots \ldots \ldots \ldots \ldots \ldots$ II cas 
La répartition n'a donc aucun caractère particulier.

e) Les autres viscères ont fait l'objet d'une étude systématique, sans qu'il soit possible de mettre en évidence des différences entre les deux lots, ni même des altérations.

L'étude de l'appareil digestif a été particulièrement soignée au niveau de l'estomac, notamment en ce qui concerne la forme et la répartition des éléments (cellules mucipares, bordantes, principales). Quelques images de nécrose ont été observées dans les deux lots au niveau des couches cellulaires superficielles de la muqueuse; elles étaient peut-être en rapport avec les techniques de sacrifice et de fixation des pièces. Ces aspects sont restés très limités. Les infiltrats inflammatoires ont été recherchés, ainsi que les aspects éventuels d'hyperplasie ou d'hypoplasie de la muqueuse.

\section{DISCUSSION}

I. - Des observations rapportées ci-dessus, il résulte qu'aucune différence n'a été observée entre les deux lots en ce qui concerne la mortalité, l'évolution pondérale, 1a consommation de nourriture, les manifestations cliniques, les formules sanguines (sauf en ce qui concerne le nombre des leucocytes), les myélogrammes, le poids de différents organes (sauf en ce qui concerne celui de la rate), l'étude anatomique des viscères.

L,es effets de l'administration prolongée de sulfites ont donc été limités :

- au cours des essais de reproduction, on observe une diminution de l'importance numérique des portées et de la proportion des mâles dans les portées ;

- à la fin de l'expérience, le nombre moyen des leucocytes contenu dans le sang est plus élevé que chez les témoins ; témoins.

- le poids moyen de la rate des femelles est également plus élevé que chez les

La signification de ces faits est d'interprétation difficile.

Les observations effectuées au cours des essais de reproduction ne se sont accompagnées apparemment d'aucune malformation chez les jeunes, dont la croissance a été d'autre part normale. L'existence bien connue, en clinique et en pathologie expérimentale, des effets de certains toxiques sur la reproduction, conduit cependant a attacher de l'importance à tout effet particulier constaté dans ce domaine. Dans le cas présent, il sera nécessaire de chercher si la diminution du nombre des jeunes par portée est due à une action sur les gamètes, à des avortements ou à une résorption embryonnaire.

Quant à l'accroissement du nombre des leucocytes dans le groupe qui a reçu du métabisulfite de potassium, il convient de souligner qu'il n'est significatif que chez les mâles ; le nombre des leucocytes dans le sang du Rat adulte varie d'ailleurs normalement entre 6 ooo et I 8 ooo par m1 (CRESKOFF et al., I962), et il suffit d'un choc émotionnel, ou d'un effort de l'animal qui se débat pendant quelques secondes pour provoquer une leucocytose. Comme on l'a vu, il reste toutefois que les cas d'hyperleucocytose avec polynucléose neutrophile, en rapport avec des lésions 
pulmonaires retrouvées à l'examen anatomique, sont un peu plus nombreux chez les animaux qui ont reçu du métabisulfite de potassium que chez les témoins.

Ennfin, l'augmentation du poids de la rate dans le groupe qui a reçu du métabisulfite de potassium ne se manifeste que chez les femelles. Un très petit nombre d'animaux en est responsable ; 1'omission de 2 d'entre eux (sur 25) suffirait à rendre non significative la différence entre les deux lots.

II. - Les résultats de notre travail concordent avec ceux d'autres études signalées au début de notre exposé mais différent de ceux obtenus par Fi'ZHUGH et al. (I946) et par FLANZY et al. (I955).

a) Rappelons que les premiers, au terme d'une triple expérimentation effectuée en trois époques différentes et en utilisant divers protocoles dont l'un visait à déterminer la part de l'avitaminose $B_{1}$ dans les troubles observés, concluaient au rôle certain des sulfites dans l'apparition des troubles suivants : polynévrite, yeux à lunettes, dépigmentation des incisives, modification de l'utérus, atrophie de différents viscères, calcification rénale tubulaire, atrophie osseuse, nécrose myocardique localisée et hyperplasie squameuse épithéliale gastrique.

Un certain nombre de lésions n'ont pas été recherchées dans notre étude : polynévrite $\left({ }^{1}\right)$, atrophie osseuse, altération de l'utérus. Mais nous n'avons pas retrouvé les autres altérations signalées par FitzHUGH et al. Plusieurs raisons peuvent être invoquées pour cela, et en particulier les différences dans les conditions d'expérience.

Nous pensons que bon nombre d'altérations signalées dans le travail de FirzHUGH et al., correspondent en fait à des modifications assez souvent rencontrées chez le rat âgé et que le nombre d'animaux utilisés, tant chez les témoins que chez les animaux traités, ne permet peut-être pas de conclure avec certitude à leur fréquence plus grande chez les animaux traités. C'est le cas en particulier pour les lésions dites de stéatose hépatique qui, dans le dernier travail de Firzhugr et al., se répartissaient comme suit :

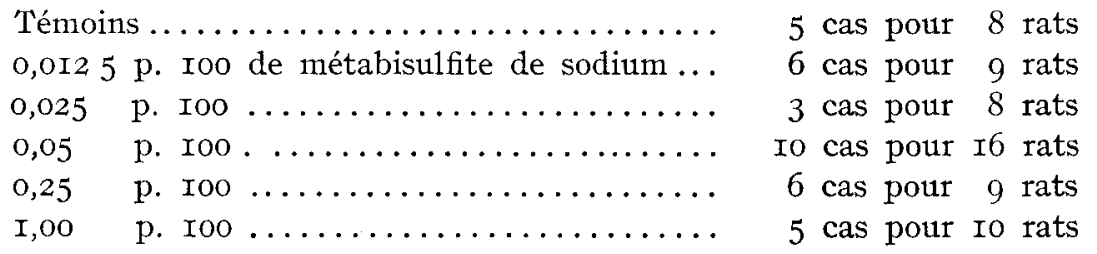

Il est vrai que pour d'autres altérations le pourcentage chez les animaux traités parait un peu plus grand que pour les témoins, mais jamais le nombre de témoins utilisé ne permet d'affirmer avec certitude que les aspects observés (assez banals en eux-même) sont valablement plus fréquents chez les animaux traités que chez les témoins.

Ennfin, dans un domaine aussi difficile que celui de l'appréciation de modifications statistiquement significatives dans les études de toxicité chronique, il serait bon de pouvoir confronter des images histologiques de ce que les auteurs considèrent comme des altérations pathologiques. Il est vrai cependant que la majorité

(1) Signalons cependant qu'aucune crise de polynévrite n'a été observée. 
des altérations signalées dans le travail de Fitzhugh et al., ne serait qu'une survenue plus fréquente, chez les animaux traités, de modifications assez courantes en ellesmêmes et ne nécessitant pas, peut-être, la présentation de micro-photographies.

b) L'absence d'effet du métabisulfite sur le développement pondéral des animaux et sur le poids du foie paraît en discordance avec les résultats que deux d'entre nous avaient obtenus antérieurement avec FLANZY (I955), résultats rappelés dans l'introduction de ce travail. Il est possible que la divergence soit due aux formes d'administration respectives des sulfites (dans le vin, ou en solution aqueuse). Mais il se peut aussi qu'elle résulte, pour une part, des conditions d'alimentation différentes dans les deux expériences: dans notre travail de I955, les animaux étaient soumis à un régime ne contenant que $2 \mathrm{mg}$ de thiamine par $\mathrm{kg}$, alors que dans la présente étude, le régime en contenait environ le double. Or, HöTzÉ (I96r-Ig62) a constaté que des doses de $\mathrm{SO}_{2}$ qui n'ont aucune action sur la croissance du Rat soumis à un régime alimentaire largement pourvu en thiamine, peuvent ralentir le développement pondéral lorsque le régime est peu riche en cette vitamine $\left(^{(}\right)$.

III. - Il y a lieu de rappeler que les doses de $\mathrm{SO}_{2}$ administrées pendant la plus grande partie de l'épreuve atteignaient approximativement $40 \mathrm{mg}$ par $\mathrm{kg}$ de poids corporel. Or, un homme de poids moyen $(65 \mathrm{~kg})$ consommant quotidiennement I litre de vin sulfité à la dose licite maximum, recevrait ainsi $7 \mathrm{mg}$ de $\mathrm{SO}_{2}$ par $\mathrm{kg}$ de poids. Le rapport entre les valeurs 40 et 7 peut paraitre élevé ; mais il est bien inférieur au rapport entre doses non toxiques pour les animaux d'expérience et doses réellement ingérées par l'Homme pour que la garantie d'innocuité puisse être jugée suffisante $\left({ }^{2}\right)$.

D'autre part, notre travail, du fait même qu'il portait sur des animaux, n'a pas permis de vérifier l'existence de certains symptômes subjectifs dont se plaint parfois le consommateur humain après ingestion d'aliments ou de boissons fortement sulfités (irritation du tractus digestif, cephalées).

Reçu pour publication en janvier 1965 .

\title{
SUMMARY
}

\author{
POTASSIUM METABISULPHITE: \\ LONG TERM STUDY OF TOXICITY IN THE RAT
}

For 20 months 2 groups of 80 rats, 40 male and 40 femela, were fed on the same balanced ration, and to drink they were given either distilled water or a solution of potassium metabisulphite, I.2 $\mathrm{g}$ per litre.

There was no difference between the groups in mortality, changes in weight or feed intake, nor in clinical signs, blood picture, except for number of white cells, or weight of different organs, except for spleen. Macroscopic and microscopic examination of viscera removed at the end of the trial, oesophagus, stomach, small intestine, colon, liver, kidneys, heart, lungs, spleen, pancreas, adrenals, sections of muscle, encephalus and trachea showed no difference between the groups.

(1) Cette constation suggère que les sulfites peuvent détruire la thiamine in vivo - - comme ils la détruisent in vitro - ou entraver son utilisation par l'organisme.

$\left.{ }^{2}\right)$ Dans le cas présent, il n'était pas possible d'expérimenter avec des solutions sulfitées beaucoup plus concentrées, qui auraient été refusées par les rats. 
The only effects seen in the animals given potassium metabisulphite were an increase in number of leucocytes, signifiant in males only, and an increase in weight of spleen, significant in females only.

On the other hand, during two successive generations the group given potassium metabisulphite produced a smaller number of young per litter, with a smaller proportion of males, than in the control group. Growth of the young, maintained in the same way as their parents, was the same in the two groups during the three months it was recorded.

The restricted nature of the differences seen between the two groups and the difficulty of interpreting them does not allow for the present any conclusion about the harmfulness of potassium metabisulphite given in the way it was. Complementary research will be necessary, particularly as regards the effects on reproduction.

\section{RÉFÉRENCES BIBLIOGRAPHIQUES}

Creskoff A. J., Frtzhugh T. jr., Farris E. J., i962. Hematology of the Rat. In Farris E. J., GrifFiTh J. Q. The Rat in laboratory investigation, p. 406-420, Hafner Publ. Co, New York.

F.A.O., I958. Réunions de la F.A.O. sur la Nutrition, Rapport $n^{0}$ 17, Rome.

Fitzhugh O. G., Knudsen L. F., Nedson A. A., I946. The chronic toxicity of sulfites. J. Pharmacol., 86, $37-48$.

Flanzy M., Causeret J., Hugot D., Guérillot J., i955. Contribution à l'étude des boissons alcooliques. II. Étude comparée de différents vins et de diverses techniques oenologiques. Ann. Technol. agric., 4, 359-380.

Gautrelet G., igro $a$. Action physiologique de l'anhydride sulfureux contenu dans les vins blancs : résumé des travaux de la Commission du Syndicat de la Propriété et du Commerce de la Gironde. Ann. Falsif., 3, 226.

GaUtrelet G., igı $b$. Recherches sur l'action physiologique de l'acide sulfureux contenu dans les vins blancs. Rapport présenté à la Commission d'Étude sur l'Ácide sulfureux, Bordeaux.

HöTzel, D., I96r. Einfluss der Versorgung mit B-Vitaminen auf die Toxicität von Schwefeldioxyd. Verhandl. Deut. Ges. inn. Med., 67, 868-871.

HöTzel D., I962. Einfluss suboptimaler Versorgung mit B-Vitaminen auf die Belastungsfähigkeit des Stoffwechsels demonstriert am Beispiel der Widerstandsfähigkeit gegen Lebensmittelfrendstoffe. Schriftenreihe des Instituts für Ernährungswissenschaft der Justus Liebig-Universität Gieszen, B. Behr's Verlag Hamburg-Berlin.

Joslyn M. A., Braverman J. B. S., I954. The chemistry and technology of the pretreatment and preser vation of fruit and vegetable products with sulfur dioxides and sulfites. Adv. Food Res., 5, 97-160.

KionKa H., I896. Ztschr. Hyg. Infekt., 22, 35r. Cité par Fitzhugh et al., i946.

Lanteaume M. T., Ramel P., Girard P., Jaulmes P., Gasc M., Ranau J., ig65. Effets physiologiques à long terme de l'anhydride sulfureux et des sulfites utilisés pour le traitement des vins rouges. Ann. Fals. 58, I6-3i.

Lehmann K. B., Treutlein A., 1909. Arch. Hyg., 68, 303-318. Cité par Truhaut, 1955.

Leuch, 1895. Korresp. bl. Schteiz. Aertze, 609. Cité par Gautrelet, $910 b$.

MiNistère DE LA SANTÉ PUBliqUE ET DE la POPULATION, 1963. Instruction du 12 septembre 1963 relative au protocole concernant les demandes d'autorisation d'emploi de substances chimiques destinées à être introduites dans les aliments et de matériaux mis au contact des aliments. Journal Officiel, 26 sept. 1963 .

Rost E., Franz F., rgr3. Arb. Kais. Gesundh., 43, 187-303. Cités par Fitzhugh et al., i946.

Schulz A, 1902. Dtsch. Med. Wochenschrift, 28, 685. Cités par Truhaut, 1955.

Truhaut R., I955. Le problème de l'introduction volontaire de substances chimiques étrangères dans les aliments : les faits, le point de vue du chimiste et du toxicologue. Bull. Soc. sci. Hyg. alim., 43, 79-1I3.

Truhaut R., ig62. Additifs aux aliments : les risques de nocivité pouvant résulter de leur emploi inconsidéré, les méthodes de prévention. Bull. Soc. Sci. Hyg. alim., 50, 77-I85.

Truhaut R., Souverain R., i963. Contrôle des substances ajoutées aux aliments en France., Coll. F. A.O. Contrôle des substances ajoutées aux aliments, no 6, 74 p., Rome.

Walbaum H., igo6. Arch. Hyg., 57, 87. Refer. Ltsch. Unters. Nahr. Genuszm., 12, 495. Cité par Truhaut, 1962.

WiLdenRath R., rgo5. Ueber die grenzen der gifligkeil des natriumsulfites. Thèse Bonn.

WILEY H. W., I907. Influence of food preservative and artificial colors on digestion and health. Sulphurous acid and sulphites. Bur. Chem. U.S. Dept. Agric. Bull., n 84 (Part. III). 